\title{
On some spaces of almost lacunary convergent sequences derived by Riesz mean and weighted almost lacunary statistical convergence in a real $n$-normed space
}

\author{
Şükran Konca* and Metin Başarır
}

"Correspondence:

skonca@sakarya.edu.tr Department of Mathematics,

Sakarya University, Sakarya, 54187, Turkey

\begin{abstract}
In this paper, we introduce some new spaces of almost convergent sequences derived by Riesz mean and the lacunary sequence in a real $n$-normed space. By combining the definitions of lacunary sequence and Riesz mean, we obtain a new concept of statistical convergence which will be called weighted almost lacunary statistical convergence in a real $n$-normed space. We examine some connections between this notion with the concept of almost lacunary statistical convergence and weighted almost statistical convergence, where the base space is a real $n$-normed space.

MSC: Primary 40C05; secondary 40A35; 46A45; 40A05; 40F05
\end{abstract}

Keywords: Riesz mean; weighted lacunary statistical convergence; almost convergence; lacunary sequence; $n$-norm

\section{Introduction}

The concept of 2-normed space has been initially introduced by Gähler [1]. Later, this concept was generalized to the concept of $n$-normed spaces by Misiak [2]. Since then, many others have studied these concepts and obtained various results [3-10].

The idea of statistical convergence was given by Zygmund [11] in 1935, in order to extend the convergence of sequences. The concept was formally introduced by Fast [12] and Steinhaus [13] and later on by Schoenberg [14], and also independently by Buck [15]. Many years later, it has been discussed in the theory of Fourier analysis, ergodic theory, and number theory under different names. In 1993, Fridy and Orhan [16] introduced the concept of lacunary statistical convergence. Statistical convergence has been generalized to the concept of a 2-normed space by Gürdal and Pehlivan [3] and to the concept of an $n$-normed space by Reddy [9].

Moricz and Orhan [17] have defined the concept of statistical summability $\left(R, p_{r}\right)$. Later on, Karakaya and Chishti [18] have used $\left(R, p_{r}\right)$-summability to generalize the concept of statistical convergence and have called this new method weighted statistical convergence. Mursaleen et al. [19] have altered the definition of weighted statistical convergence and have found its relation with the concept of statistical $\left(R, p_{r}\right)$-summability. In general, the statistical convergence of weighted mean is studied as a regular matrix transformation. In

○2014 Konca and Başarı; licensee Springer. This is an Open Access article distributed under the terms of the Creative Commons Attribution License (http://creativecommons.org/licenses/by/2.0), which permits unrestricted use, distribution, and reproduction in any medium, provided the original work is properly cited. 
[18] and [19], the concept of statistical convergence is generalized by using a Riesz summability method and it is called weighted statistical convergence. For more details related to this topic, we may refer to [5, 20-23].

In this paper, we introduce some new spaces of almost convergent sequences derived by Riesz mean and lacunary sequence in a real $n$-normed space. By combining the definitions of lacunary sequence and Riesz mean, we obtain a new concept of statistical convergence, which will be called weighted almost lacunary statistical convergence in a real $n$-normed space. We examine some connections between this notion with the concept of almost lacunary statistical convergence and weighted almost statistical convergence, where the base space is a real $n$-normed space.

\section{Definitions and preliminaries}

Let $K$ be a subset of natural numbers $\mathbb{N}$ and we denote the set $K_{n}=\{j \in K: j \leq n\}$. The cardinality of $K_{n}$ is denoted by $\left|K_{n}\right|$. The natural density of $K$ is given by $\delta(K):=\lim _{r} \frac{1}{r}\left|K_{r}\right|$, if it exists. The sequence $x=\left(x_{j}\right)$ is statistically convergent to $\xi$ provided that, for every $\varepsilon>0$, the set $K=K(\varepsilon):=\left\{j \in \mathbb{N}:\left|x_{j}-\xi\right| \geq \varepsilon\right\}$ has natural density zero.

Let $\left(p_{k}\right)$ be a sequence of non-negative real numbers and $P_{r}=p_{1}+p_{2}+\cdots+p_{r}$ for $r \in \mathbb{N}$. Then the Riesz transformation of $x=\left(x_{k}\right)$ is defined as

$$
t_{r}:=\frac{1}{P_{r}} \sum_{k=1}^{r} p_{k} x_{k} .
$$

If the sequence $t_{r}$ has a finite limit $\xi$, then the sequence $x$ is said to be $\left(R, p_{r}\right)$-convergent to $\xi$. Let us note that if $P_{r} \rightarrow \infty$ as $r \rightarrow \infty$ then the Riesz transformation is a regular summability method, that is, it transforms every convergent sequence to convergent sequence and preserves the limit.

If $p_{k}=1$ for all $k \in \mathbb{N}$ in (2.1), then the Riesz mean reduces to the Cesaro mean $C_{1}$ of order one.

By a lacunary sequence $\theta=\left(k_{r}\right)$, where $k_{0}=0$, we will mean an increasing sequence of non-negative integers with $k_{r}-k_{r-1} \rightarrow \infty$ as $r \rightarrow \infty$. The intervals determined by $\theta$ will be denoted by $I_{r}=\left(k_{r-1}, k_{r}\right]$. We write $h_{r}=k_{r}-k_{r-1}$. The ratio $\frac{k_{r}}{k_{r-1}}$ will be denoted by $q_{r}$.

Throughout the paper, we will use the following notations, which have been defined in [24].

Let $\theta=\left(k_{r}\right)$ be a lacunary sequence, $\left(p_{k}\right)$ be a sequence of positive real numbers such that $H_{r}:=\sum_{k \in I_{r}} p_{k}, P_{k_{r}}:=\sum_{k \in\left(0, k_{r}\right]} p_{k}, P_{k_{r-1}}:=\sum_{k \in\left(0, k_{r-1}\right]} p_{k}, Q_{r}:=\frac{P_{k_{r}}}{P_{k_{r}-1}}, P_{0}=0$ and the intervals determined by $\theta$ and $\left(p_{k}\right)$ are denoted by $I_{r}^{\prime}=\left(P_{k_{r-1}}, P_{k_{r}}\right], H_{r}=P_{k_{r}}-P_{k_{r-1}}$. If $p_{k}=1$ for all $k \in \mathbb{N}$, then $H_{r}, P_{k_{r}}, P_{k_{r-1}}, Q_{r}$ and $I_{r}^{\prime}$ reduce to $h_{r}, k_{r}, k_{r-1}, q_{r}$ and $I_{r}$, respectively.

If $\theta=\left(k_{r}\right)$ is a lacunary sequence and $P_{r} \rightarrow \infty$ as $r \rightarrow \infty$, then $\theta^{\prime}=\left(P_{k_{r}}\right)$ is a lacunary sequence, that is, $P_{0}=0,0<P_{k_{r-1}}<P_{k_{r}}$ and $H_{r}=P_{k_{r}}-P_{k_{r-1}} \rightarrow \infty$ as $r \rightarrow \infty$.

Throughout the paper, we will take $P_{r} \rightarrow \infty$ as $r \rightarrow \infty$, unless otherwise stated.

Lorentz [25] has proved that a sequence $x$ is almost convergent to a number $\xi$ if and only if $t_{k m}(x) \rightarrow \xi$ as $k \rightarrow \infty$, uniformly in $m$, where

$$
t_{k m}(x)=\frac{x_{m}+x_{m+1}+\cdots+x_{m+k-1}}{k}, \quad k \in \mathbb{N}, m \geq 0 .
$$


We write $f-\lim x=\xi$ if $x$ is almost convergent to $\xi$. Maddox [26] has defined $x=\left(x_{j}\right)$ to be strongly almost convergent to a number $\xi$ if and only if $t_{k m}(|x-\xi e|) \rightarrow 0$ as $k \rightarrow \infty$, uniformly in $m$, where $x-\xi e=\left(x_{j}-\xi\right)$ for all $j$ and $e=(1,1, \ldots)$.

Let $n \in \mathbb{N}$ and $X$ be a real vector space of dimension $d \geq n \geq 2$. A real-valued function $\|\cdot, \ldots, \cdot\|: X^{n} \rightarrow \mathbb{R}$ satisfying the following conditions is called an $n$-norm on $X$ and the pair $(X,\|\cdot, \ldots, \cdot\|)$ is called a linear $n$-normed space:

(1) $\left\|x_{1}, \ldots, x_{n}\right\|=0$ if and only if $x_{1}, \ldots, x_{n}$ are linearly dependent,

(2) $\left\|x_{1}, \ldots, x_{n}\right\|$ is invariant under permutation,

(3) $\left\|\alpha x_{1}, \ldots, x_{n-1}, x_{n}\right\|=|\alpha|\left\|x_{1}, \ldots, x_{n-1}, x_{n}\right\|$ for any $\alpha \in \mathbb{R}$,

(4) $\left\|x_{1}, \ldots, x_{n-1}, y+z\right\| \leq\left\|x_{1}, \ldots, x_{n-1}, y\right\|+\left\|x_{1}, \ldots, x_{n-1}, z\right\|$, for all $y, z, x_{1}, \ldots, x_{n-1} \in X$.

A sequence $x=\left(x_{j}\right)$ in an $n$-normed space $(X,\|\cdot, \ldots, \cdot\|)$ is said to be convergent to some $\xi \in X$ in the $n$-norm if for each $\varepsilon>0$ there exists a positive integer $j_{0}=j_{0}(\varepsilon)$ such that $\left\|x_{j}-\xi, z_{1}, \ldots, z_{n-1}\right\|<\varepsilon$ for all $j \geq j_{0}$ and for every nonzero $z_{1}, \ldots, z_{n-1} \in X$.

A sequence $x=\left(x_{j}\right)$ is said to be statistically convergent to $\xi$ if for every $\varepsilon>0$ the set $K:=$ $\left\{j \in \mathbb{N}:\left\|x_{j}-\xi, z_{1}, \ldots, z_{n-1}\right\| \geq \varepsilon\right\}$ has natural density zero for every nonzero $z_{1}, \ldots, z_{n-1} \in X$, in other words, $x=\left(x_{j}\right)$ is statistically convergent to $\xi$ in $n$-normed space $(X,\|\cdot, \ldots, \cdot\|)$ if $\lim _{j \rightarrow \infty} \frac{1}{j}\left|\left\{j \in \mathbb{N}:\left\|x_{j}-\xi, z_{1}, \ldots, z_{n-1}\right\| \geq \varepsilon\right\}\right|=0$, for every nonzero $z_{1}, \ldots, z_{n-1} \in X$. For $\xi=0$, we say this is statistically null.

\section{Main results}

Throughout the paper $w(X), l_{\infty}(X)$ denote the spaces of all and bounded $X$ valued sequence spaces, respectively, where $(X,\|\cdot, \ldots, \cdot\|)$ is a real $n$-normed space.

The set of all almost convergent sequences and strongly almost convergent sequences with respect to the $n$-norm $\|\cdot, \cdot\|$ are denoted by $F$ and $[F]$, respectively, as follows:

$$
F=\left\{\begin{array}{l}
x \in l_{\infty}(X): \lim _{k \rightarrow \infty}\left\|t_{k m}(x-\xi e), z_{1}, \ldots, z_{n-1}\right\|=0, \text { uniformly in } m, \\
\text { for every nonzero } z_{1}, \ldots, z_{n-1} \in X
\end{array}\right\},
$$

and

$$
[F]=\left\{\begin{array}{l}
x \in l_{\infty}(X): \lim _{k \rightarrow \infty} t_{k m}\left(\left\|x-\xi e, z_{1}, \ldots, z_{n-1}\right\|\right)=0, \text { uniformly in } m, \\
\text { for every nonzero } z_{1}, \ldots, z_{n-1} \in X
\end{array}\right\}
$$

where $t_{k m}(x)$ is defined as in (2.2). We write $F-\lim x=\xi$ if $x$ is almost convergent to $\xi$ with respect to the $n$-norm and $[F]-\lim x=\xi$ if $x$ is strongly almost convergent to $\xi$ with respect to the $n$-norm. It is easy to see that the inclusions $[F] \subset F \subset l \infty(X)$ hold.

Now, we define some new sequence spaces in a real $n$-normed space as follows:

$$
\begin{aligned}
& {\left[\tilde{R}, p_{r}, \theta\right]_{n}=\left\{\begin{array}{l}
x: \lim _{r \rightarrow \infty}\left\|\frac{1}{H_{r}} \sum_{k \in I_{r}} p_{k} t_{k m}(x-\xi e), z_{1}, \ldots, z_{n-1}\right\|=0, \text { uniformly in } m, \\
\text { for some } \xi \text { and for every nonzero } z_{1}, \ldots, z_{n-1} \in X
\end{array}\right\},} \\
& \left(\tilde{R}, p_{r}, \theta\right)_{n}=\left\{\begin{array}{l}
x: \lim _{r \rightarrow \infty} \frac{1}{H_{r}} \sum_{k \in I_{r}} p_{k}\left\|t_{k m}(x-\xi e), z_{1}, \ldots, z_{n-1}\right\|=0, \text { uniformly in } m, \\
\text { for some } \xi \text { and for every nonzero } z_{1}, \ldots, z_{n-1} \in X
\end{array}\right\}, \\
& \left|\tilde{R}, p_{r}, \theta\right|_{n}=\left\{\begin{array}{l}
x: \lim _{r \rightarrow \infty} \frac{1}{H_{r}} \sum_{k \in I_{r}} p_{k} t_{k m}\left(\left\|x-\xi e, z_{1}, \ldots, z_{n-1}\right\|\right)=0, \text { uniformly in } m, \\
\text { for some } \xi \text { and for every nonzero } z_{1}, \ldots, z_{n-1} \in X
\end{array}\right\} .
\end{aligned}
$$

The following results are obtained for some special cases: 
(1) If we take $m=0$ then the sequence spaces above are reduced to the sequence spaces $\left[C_{1}, \theta\right]_{n},\left(C_{1}, \theta\right)_{n},\left|C_{1}, \theta\right|_{n}$, respectively as follows:

$$
\begin{aligned}
{\left[C_{1}, \theta\right]_{n} } & =\left\{\begin{array}{l}
x: \lim _{r \rightarrow \infty}\left\|\frac{1}{H_{r}} \sum_{k \in I_{r}} p_{k} t_{k 0}(x-\xi e), z_{1}, \ldots, z_{n-1}\right\|=0, \\
\text { for some } \xi \text { and for every nonzero } z_{1}, \ldots, z_{n-1} \in X
\end{array}\right\}, \\
\left(C_{1}, \theta\right)_{n} & =\left\{\begin{array}{l}
x: \lim _{r \rightarrow \infty} \frac{1}{H_{r}} \sum_{k \in I_{r}} p_{k}\left\|t_{k 0}(x-\xi e), z_{1}, \ldots, z_{n-1}\right\|=0 \\
\text { for some } \xi \text { and for every nonzero } z_{1}, \ldots, z_{n-1} \in X
\end{array}\right\}, \\
\left|C_{1}, \theta\right|_{n} & =\left\{\begin{array}{l}
x: \lim _{r \rightarrow \infty} \frac{1}{H_{r}} \sum_{k \in I_{r}} p_{k} t_{k 0}\left\|x-\xi e, z_{1}, \ldots, z_{n-1}\right\|=0, \\
\text { for some } \xi \text { and for every nonzero } z_{1}, \ldots, z_{n-1} \in X
\end{array}\right\} .
\end{aligned}
$$

(2) If we take $p_{k}=1$ for all $k \in \mathbb{N}$, then the sequence spaces above are reduced to the following spaces:

$$
\begin{aligned}
{\left[w_{\theta}\right]_{n} } & =\left\{\begin{array}{l}
x: \lim _{r \rightarrow \infty}\left\|\frac{1}{h_{r}} \sum_{k \in I_{r}} t_{k m}(x-\xi e), z_{1}, \ldots, z_{n-1}\right\|=0, \text { uniformly in } m, \\
\text { for some } \xi \text { and for every nonzero } z_{1}, \ldots, z_{n-1} \in X
\end{array}\right\}, \\
\left(w_{\theta}\right)_{n} & =\left\{\begin{array}{l}
x: \lim _{r \rightarrow \infty} \frac{1}{h_{r}} \sum_{k \in I_{r}}\left\|t_{k m}(x-\xi e), z_{1}, \ldots, z_{n-1}\right\|=0, \text { uniformly in } m, \\
\text { for some } \xi \text { and for every nonzero } z_{1}, \ldots, z_{n-1} \in X
\end{array}\right\}, \\
\left|w_{\theta}\right|_{n} & =\left\{\begin{array}{l}
x: \lim _{r \rightarrow \infty} \frac{1}{h_{r}} \sum_{k \in I_{r}} t_{k m}\left(\left\|x-\xi e, z_{1}, \ldots, z_{n-1}\right\|\right)=0, \text { uniformly in } m, \\
\text { for some } \xi \text { and for every nonzero } z_{1}, \ldots, z_{n-1} \in X
\end{array}\right\} .
\end{aligned}
$$

(3) Let us choose $\theta=\left(k_{r}\right)=2^{r}$ for $r>0$, then these sequence spaces above are reduced to the following spaces:

$$
\begin{aligned}
{\left[\tilde{R}, p_{r}\right]_{n} } & =\left\{\begin{array}{l}
x: \lim _{r \rightarrow \infty}\left\|\frac{1}{P_{r}} \sum_{k=1}^{r} p_{k} t_{k m}(x-\xi e), z_{1}, \ldots, z_{n-1}\right\|=0, \text { uniformly } \\
\text { in } m, \text { for some } \xi \text { and for every nonzero } z_{1}, \ldots, z_{n-1} \in X
\end{array}\right\}, \\
\left(\tilde{R}, p_{r}\right)_{n} & =\left\{\begin{array}{l}
x: \lim _{r \rightarrow \infty} \frac{1}{P_{r}} \sum_{k=1}^{r} p_{k}\left\|t_{k m}(x-\xi e), z_{1}, \ldots, z_{n-1}\right\|=0, \text { uniformly } \\
\text { in } m, \text { for some } \xi \text { and for every nonzero } z_{1}, \ldots, z_{n-1} \in X
\end{array}\right\}, \\
\left|\tilde{R}, p_{r}\right|_{n} & =\left\{\begin{array}{l}
x: \lim _{r \rightarrow \infty} \frac{1}{P_{r}} \sum_{k=1}^{r} p_{k} t_{k m}\left(\left\|x-\xi e, z_{1}, \ldots, z_{n-1}\right\|\right)=0, \text { uniformly } \\
\text { in } m, \text { for some } \xi \text { and for every nonzero } z_{1}, \ldots, z_{n-1} \in X
\end{array}\right\} .
\end{aligned}
$$

(4) If we select $\theta=\left(k_{r}\right)=2^{r}$ for $r>0$ and the base space as $(X,\|\cdot, \cdot\|)$ then these sequence spaces above are reduced to the sequence spaces which can be seen in [5].

(5) If we choose $p_{k}=1$ for all $k \in \mathbb{N}$ and $\theta=\left(k_{r}\right)=2^{r}$ for $r>0$, then these sequence spaces above are reduced to the sequence spaces $\left[C_{1}\right]_{n},\left(C_{1}\right)_{n},\left|C_{1}\right|_{n}$, respectively.

Now, we give the following theorem to demonstrate some inclusion relations among the sequence spaces $\left|\tilde{R}, p_{r}, \theta\right|_{n},\left(\tilde{R}, p_{r}, \theta\right)_{n},\left[\tilde{R}, p_{r}, \theta\right]_{n},\left|C_{1}, \theta\right|_{n},\left(C_{1}, \theta\right)_{n},\left[C_{1}, \theta\right]_{n}$ with the spaces $F$ and $[F]$.

Theorem 3.1 The following statements are true:

(1) $[F] \subset F \subset\left(\tilde{R}, p_{r}, \theta\right)_{n} \subset\left[\tilde{R}, p_{r}, \theta\right]_{n} \subset\left[C_{1}, \theta\right]_{n}$.

(2) $[F] \subset\left|\tilde{R}, p_{r}, \theta\right|_{n} \subset\left(\tilde{R}, p_{r}, \theta\right)_{n} \subset\left[\tilde{R}, p_{r}, \theta\right]_{n} \subset\left[C_{1}, \theta\right]_{n}$.

(3) $[F] \subset\left|\tilde{R}, p_{r}, \theta\right|_{n} \subset\left|C_{1}, \theta\right| \subset\left(C_{1}, \theta\right)_{n} \subset\left[C_{1}, \theta\right]_{n}$. 
Proof We give the proof only for (2). The proofs of (1) and (3) can be done, similarly. So we omit them. Let $x \in[F]$ and $[F]-\lim x=\xi$. Then $t_{k m}\left(\left\|x-\xi e, z_{1}, \ldots, z_{n-1}\right\|\right) \rightarrow 0$ as $k \rightarrow \infty$, uniformly in $m$, for every nonzero $z_{1}, \ldots, z_{n-1} \in X$. Since $H_{r} \rightarrow \infty$ as $r \rightarrow \infty$, then its weighted lacunary mean also converges to $\xi$ as $r \rightarrow \infty$ uniformly in $m$. This proves that $x \in\left|\tilde{R}, p_{r}, \theta\right|_{n}$ and $[F]-\lim x=\left|\tilde{R}, p_{r}, \theta\right|_{n}-\lim x=\xi$. Also since

$$
\begin{aligned}
\left\|\frac{1}{H_{r}} \sum_{k \in I_{r}} p_{k} t_{k m}(x-\xi e), z_{1}, \ldots, z_{n-1}\right\| & \leq \frac{1}{H_{r}} \sum_{k \in I_{r}} p_{k}\left\|t_{k m}(x-\xi e), z_{1}, \ldots, z_{n-1}\right\| \\
& \leq \frac{1}{H_{r}} \sum_{k \in I_{r}} p_{k} t_{k m}\left(\left\|x-\xi e, z_{1}, \ldots, z_{n-1}\right\|\right),
\end{aligned}
$$

then it follows that $[F] \subset\left|\tilde{R}, p_{r}, \theta\right|_{n} \subset\left(\tilde{R}, p_{r}, \theta\right)_{n} \subset\left[\tilde{R}, p_{r}, \theta\right]_{n}$ and $[F]-\lim x=\left|\tilde{R}, p_{r}, \theta\right|_{n}-$ $\lim x=\left(\tilde{R}, p_{r}, \theta\right)_{n}-\lim x=\left[\tilde{R}, p_{r}, \theta\right]_{n}-\lim x=\xi$. Since uniform convergence of $\| \frac{1}{H_{r}} \times$ $\sum_{k \in I_{r}} p_{k} t_{k m}(x-\xi e), z_{1}, \ldots, z_{n-1} \|$ with respect to $m$, as $r \rightarrow \infty$, implies convergence for $m=0$ and for every nonzero $z_{1}, \ldots, z_{n-1} \in X$. It follows that $\left[\tilde{R}, p_{r}, \theta\right]_{n} \subset\left[C_{1}, \theta\right]_{n}$ and $\left[\tilde{R}, p_{r}, \theta\right]_{n}-\lim x=\left[C_{1}, \theta\right]_{n}-\lim x=\xi$. This completes the proof.

Theorem 3.2 Let $\theta=\left(k_{r}\right)$ be a lacunary sequence and $\liminf _{r} Q_{r}>1$. Then $\left(\tilde{R}, p_{r}\right)_{n} \subseteq$ $\left(\tilde{R}, p_{r}, \theta\right)_{n}$ with $\left(\tilde{R}, p_{r}\right)_{n}-\lim x=\left(\tilde{R}, p_{r}, \theta\right)_{n}-\lim x=\xi$.

Proof Suppose that $\liminf _{r} Q_{r}>1$, then there exists a $\delta>0$ such that $Q_{r} \geq 1+\delta$ for sufficiently large values of $r$, which implies that $\frac{H_{r}}{P_{k_{r}}} \geq \frac{\delta}{1+\delta}$. If $x \in\left(\tilde{R}, p_{r}\right)_{n}$ with $\left(\tilde{R}, p_{r}\right)_{n}-\lim x=\xi$, then for sufficiently large values of $r$, we have

$$
\begin{aligned}
& \frac{1}{P_{k_{r}}} \sum_{k=1}^{k_{r}} p_{k}\left\|t_{k m}(x-\xi e), z_{1}, \ldots, z_{n-1}\right\| \\
& \quad=\frac{1}{P_{k_{r}}}\left(\sum_{k=1}^{k_{r-1}} p_{k}\left\|t_{k m}(x-\xi e), z_{1}, \ldots, z_{n-1}\right\|+\sum_{k=k_{r-1}+1}^{k_{r}} p_{k}\left\|t_{k m}(x-\xi e), z_{1}, \ldots, z_{n-1}\right\|\right) \\
& \quad \geq \frac{H_{r}}{P_{k_{r}}}\left(\frac{1}{H_{r}} \sum_{k \in I_{r}} p_{k}\left\|t_{k m}(x-\xi e), z_{1}, \ldots, z_{n-1}\right\|\right) \\
& \quad \geq \frac{\delta}{1+\delta} \cdot \frac{1}{H_{r}} \sum_{k \in I_{r}} p_{k}\left\|t_{k m}(x-\xi e), z_{1}, \ldots, z_{n-1}\right\|,
\end{aligned}
$$

for each $m \geq 0$ and for every nonzero $z_{1}, \ldots, z_{n-1} \in X$. Then, it follows that $x \in\left(\tilde{R}, p_{r}, \theta\right)_{n}$ with $\left(\tilde{R}, p_{r}, \theta\right)_{n}-\lim x=\xi$ by taking the limit as $r \rightarrow \infty$. This completes the proof.

Theorem 3.3 Let $\theta=\left(k_{r}\right)$ be a lacunary sequence with $\lim \sup _{r} Q_{r}<\infty$. Then $\left(\tilde{R}, p_{r}, \theta\right)_{n} \subseteq$ $\left(\tilde{R}, p_{r}\right)_{n}$ with $\left(\tilde{R}, p_{r}, \theta\right)_{n}-\lim x=\left(\tilde{R}, p_{r}\right)_{n}-\lim x=\xi$.

Proof Let $x \in\left(\tilde{R}, p_{r}, \theta\right)_{n}$ with $\left(\tilde{R}, p_{r}, \theta\right)_{n}-\lim x=\xi$. Then for $\varepsilon>0$, there exists $q_{0}$ such that for every $q>q_{0}$

$$
L_{q}=\frac{1}{H_{q}} \sum_{k \in I_{q}} p_{k}\left\|t_{k m}(x-\xi e), z_{1}, \ldots, z_{n-1}\right\|<\varepsilon,
$$


for each $m \geq 0$ and for every nonzero $z_{1}, \ldots, z_{n-1} \in X$, that is, we can find some positive constant $M$ such that

$$
L_{q} \leq M \text { for all } q \text {. }
$$

$\limsup \operatorname{su}_{r}<\infty$ implies that there exists some positive number $K$ such that

$$
Q_{r} \leq K \quad \text { for all } r \geq 1 \text {. }
$$

Therefore for $k_{r-1}<r \leq k_{r}$, we have by (3.1), (3.2), and (3.3)

$$
\begin{aligned}
& \frac{1}{P_{r}} \sum_{k=1}^{r} p_{k}\left\|t_{k m}(x-\xi e), z_{1}, \ldots, z_{n-1}\right\| \\
& \leq \frac{1}{P_{k_{r-1}}} \sum_{k=1}^{k_{r}} p_{k}\left\|t_{k m}(x-\xi e), z_{1}, \ldots, z_{n-1}\right\| \\
&=\frac{1}{P_{k_{r-1}}}\left(\sum_{k \in I_{1}} p_{k}\left\|t_{k m}(x-\xi e), z_{1}, \ldots, z_{n-1}\right\|+\sum_{k \in I_{2}} p_{k}\left\|t_{k m}(x-\xi e), z_{1}, \ldots, z_{n-1}\right\|+\cdots\right. \\
&\left.\quad+\sum_{k \in I_{q_{0}}} p_{k}\left\|t_{k m}(x-\xi e), z_{1}, \ldots, z_{n-1}\right\|+\cdots+\sum_{k \in I_{r}} p_{k}\left\|t_{k m}(x-\xi e), z_{1}, \ldots, z_{n-1}\right\|\right) \\
&=\frac{1}{P_{k_{r-1}}}\left(L_{1} H_{1}+L_{2} H_{2}+\cdots+L_{q_{0}} H_{q_{0}}+L_{q_{0}+1} H_{q_{0}+1}+\cdots+L_{r} H_{r}\right) \\
& \leq \frac{M}{P_{k_{r-1}}}\left(H_{1}+H_{2}+\cdots+H_{q_{0}}\right)+\frac{\varepsilon}{P_{k_{r-1}}}\left(H_{q_{0}+1}+\cdots+H_{r}\right) \\
&=\frac{M}{P_{k_{r-1}}}\left(P_{k_{1}}-P_{k_{0}}+\cdots+P_{k_{q_{0}}}-P_{k_{q_{0}-1}}\right)+\frac{\varepsilon}{P_{k_{r-1}}}\left(P_{k_{q_{0}}}-P_{k_{q_{0}-1}}+\cdots+P_{k_{r}}-P_{k_{r-1}}\right) \\
&= M \frac{P_{k_{q_{0}}}}{P_{k_{r-1}}}+\varepsilon \frac{P_{k_{r}}-P_{k_{q_{0}}}}{P_{k_{r-1}}} \\
& \leq M \frac{P_{k_{q_{0}}}}{P_{k_{r-1}}}+\varepsilon K
\end{aligned}
$$

for each $m \geq 0$ and for every nonzero $z_{1}, \ldots, z_{n-1} \in X$. Since $P_{k_{r-1}} \rightarrow \infty$ as $r \rightarrow \infty$, we get $x \in\left(\tilde{R}, p_{r}\right)_{n}$ with $\left(\tilde{R}, p_{r}\right)_{n}-\lim x=\xi$. This completes the proof.

Corollary 3.4 Let $1<\liminf _{r} Q_{r} \leq \limsup \sup _{r} Q_{r}<$. Then $\left(\tilde{R}, p_{r}, \theta\right)_{n}=\left(\tilde{R}, p_{r}\right)_{n}$ and $\left(\tilde{R}, p_{r}, \theta\right)_{n}-\lim x=\left(\tilde{R}, p_{r}\right)_{n}-\lim x=\xi$.

Proof It follows from Theorem 3.2 and Theorem 3.3.

In the following theorem, we give the relations between the sequence spaces $\left(w_{\theta}\right)_{n}$ and $\left(\tilde{R}, p_{r}\right)_{n}$.

\section{Theorem 3.5}

(1) If $p_{k}<1$ for all $k \in \mathbb{N}$, then $\left(w_{\theta}\right)_{n} \subseteq\left(\tilde{R}, p_{r}\right)_{n}$ and $\left(w_{\theta}\right)_{n}-\lim x=\left(\tilde{R}, p_{r}\right)_{n}-\lim x=\xi$.

(2) If $p_{k}>1$ for all $k \in \mathbb{N}$ and $\left(\frac{H_{r}}{h_{r}}\right)$ is upper-bounded, then $\left(\tilde{R}, p_{r}\right)_{n} \subseteq\left(w_{\theta}\right)_{n}$ and $\left(\tilde{R}, p_{r}\right)_{n}-\lim x=\left(w_{\theta}\right)_{n}-\lim x=\xi$. 
(1) If $p_{k}<1$ for all $k \in \mathbb{N}$, then $H_{r}<h_{r}$ for all $r \in \mathbb{N}$. So, there exists an $M_{1}$, a constant, such that $0<M_{1} \leq \frac{H_{r}}{h_{r}}<1$ for all $r \in \mathbb{N}$. Let $x \in\left(w_{\theta}\right)_{n}$ with $\left(w_{\theta}\right)_{n}-\lim x=\xi$, then for an arbitrary $\varepsilon>0$ we have

$$
\frac{1}{H_{r}} \sum_{k \in I_{r}} p_{k}\left\|t_{k m}(x-\xi e), z_{1}, \ldots, z_{n-1}\right\| \leq \frac{1}{M_{1}} \frac{1}{h_{r}} \sum_{k \in I_{r}}\left\|t_{k m}(x-\xi e), z_{1}, \ldots, z_{n-1}\right\|,
$$

for each $m \geq 0$ and for every nonzero $z_{1}, \ldots, z_{n-1} \in X$. Therefore, we get the result by taking the limit as $r \rightarrow \infty$.

(2) Let $p_{k}>1$ for all $k \in \mathbb{N}$, then $H_{r}>h_{r}$ for all $r \in \mathbb{N}$. Suppose that $\left(\frac{H_{r}}{h_{r}}\right)$ is upper-bounded, then there exists an $M_{2}$, a constant, such that $1<\frac{H_{r}}{h_{r}} \leq M_{2}<\infty$ for all $r \in \mathbb{N}$. Let $x \in\left(\tilde{R}, p_{r}\right)_{n}$ and $\left(\tilde{R}, p_{r}\right)_{n}-\lim x=\xi$. So the result is obtained by taking the limit as $r \rightarrow \infty$ for each $m \geq 0$ and for every nonzero $z_{1}, \ldots, z_{n-1} \in X$, from the following inequality:

$$
\frac{1}{h_{r}} \sum_{k \in I_{r}}\left\|t_{k m}(x-\xi e), z_{1}, \ldots, z_{n-1}\right\| \leq M_{2} \frac{1}{H_{r}} \sum_{k \in I_{r}} p_{k}\left\|t_{k m}(x-\xi e), z_{1}, \ldots, z_{n-1}\right\| .
$$

Now, we define a new concept of statistical convergence in $n$-normed space, which will be called weighted almost lacunary statistical convergence:

Definition 3.6 The weighted almost lacunary density of $K \subseteq \mathbb{N}$ is denoted by $\delta_{(\tilde{R}, \theta)}(K)=$ $\lim _{r \rightarrow \infty} \frac{1}{H_{r}}\left|K_{r}(\varepsilon)\right|$ if the limit exists. We say that the sequence $x=\left(x_{j}\right)$ is weighted almost lacunary statistically convergent to $\xi$ if for every $\varepsilon>0$, the set $K_{r}(\varepsilon)=\left\{k \in I_{r}^{\prime}\right.$ : $\left.p_{k}\left\|t_{k m}(x-\xi e), z_{1}, \ldots, z_{n-1}\right\| \geq \varepsilon\right\}$ has weighted lacunary density zero, i.e.

$$
\lim _{r \rightarrow \infty} \frac{1}{H_{r}}\left|\left\{k \in I_{r}^{\prime}: p_{k}\left\|t_{k m}(x-\xi e), z_{1}, \ldots, z_{n-1}\right\| \geq \varepsilon\right\}\right|=0
$$

uniformly in $m$, for every nonzero $z_{1}, \ldots, z_{n-1} \in X$. In this case, we write $\left(S_{(\tilde{R}, \theta)}, n\right)-\lim _{k} x_{k}=$ $\xi$. By $\left(S_{(\tilde{R}, \theta)}, n\right)$ we denote the set of all weighted almost lacunary statistically convergent sequences in $n$-normed space.

(1) If we take $p_{k}=1$ for all $k \in \mathbb{N}$ in (3.4) then we obtain the definition of almost lacunary statistical convergence in $n$-normed space, that is, $x$ is called almost lacunary statistically convergent to $\xi$ if for every $\varepsilon>0$, the set $K_{\theta}(\varepsilon)=\left\{k \in I_{r}:\left\|t_{k m}(x-\xi e), z_{1}, \ldots, z_{n-1}\right\| \geq \varepsilon\right\}$ has lacunary density zero, i.e.

$$
\lim _{r \rightarrow \infty} \frac{1}{h_{r}}\left|\left\{k \in I_{r}:\left\|t_{k m}(x-\xi e), z_{1}, \ldots, z_{n-1}\right\| \geq \varepsilon\right\}\right|=0
$$

uniformly in $m$, for every nonzero $z_{1}, \ldots, z_{n-1} \in X$. In this case, we write $\left(S_{\theta}, n\right)-\lim _{j} x_{j}=\xi$. By $\left(S_{\theta}, n\right)$ we denote the set of all weighted almost lacunary statistically convergent sequences in $n$-normed space.

(2) Let us choose $\theta=\left(k_{r}\right)$ for $r>0$ then the definition of weighted almost lacunary statistical convergence which is given in (3.4) is reduced to the definition of weighted almost statistically convergence, that is, $x$ is called weighted almost 
statistically convergent to $\xi$ if for every $\varepsilon>0$, the set

$K_{P_{r}}(\varepsilon)=\left\{k \leq P_{r}: p_{k}\left\|t_{k m}(x-\xi e), z_{1}, \ldots, z_{n-1}\right\| \geq \varepsilon\right\}$ has weighted density zero, i.e.

$$
\lim _{r \rightarrow \infty} \frac{1}{P_{r}}\left|\left\{k \leq P_{r}:\left\|t_{k m}(x-\xi e), z_{1}, \ldots, z_{n-1}\right\| \geq \varepsilon\right\}\right|=0
$$

uniformly in $m$, for every nonzero $z_{1}, \ldots, z_{n-1} \in X$. In this case, we write $\left(S_{\tilde{R}}, n\right)-\lim _{j} x_{j}=\xi$. By $\left(S_{\tilde{R}}, n\right)$ we denote the set of all weighted almost lacunary statistically convergent sequences in $n$-normed space.

(3) Let us choose $\theta=\left(k_{r}\right)$ for $r>0$ and $p_{k}=1$ for all $k \in \mathbb{N}$, then the definition of weighted almost lacunary statistical convergence, which is given in (3.4), is reduced to the definition of almost statistical convergence.

Theorem 3.7 If the sequence $x$ is $\left(\tilde{R}, p_{r}, \theta\right)_{n}$-convergent to $\xi$ then the sequence $x$ is weighted almost lacunary statistically convergent to $\xi$.

Proof Let the sequence $x$ be $\left(\tilde{R}, p_{r}, \theta\right)_{n}$-convergent to $\xi$ and $K_{r m}(\varepsilon)=\left\{k \in I_{r}^{\prime}: p_{k} \| t_{k m}(x-\right.$ $\left.\xi e), z_{1}, \ldots, z_{n-1} \| \geq \varepsilon\right\}$. Then for a given $\varepsilon>0$, we have

$$
\begin{aligned}
\frac{1}{H_{r}} \sum_{k \in I_{r}} p_{k}\left\|t_{k m}(x-\xi e), z_{1}, \ldots, z_{n-1}\right\| & \geq \frac{1}{H_{r}} \sum_{\substack{k \in I_{r} \\
k \in K_{r m}(\varepsilon)}} p_{k}\left\|t_{k m}(x-\xi e), z_{1}, \ldots, z_{n-1}\right\| \\
& \geq \varepsilon \frac{1}{H_{r}}\left|K_{r m}(\varepsilon)\right|
\end{aligned}
$$

for each $m \geq 0$ and for every nonzero $z \in X$. Hence, we see that the sequence $x$ is weighted almost statistically convergent to $\xi$ by taking the limit as $r \rightarrow \infty$.

Theorem 3.8 Let $p_{k}\left\|t_{k m}(x-\xi e), z_{1}, \ldots, z_{n-1}\right\| \leq M$ for all $k \in \mathbb{N}$, for each $m \geq 0$ and for every nonzero $z_{1}, \ldots, z_{n-1} \in X$. Then $\left(S_{(\tilde{R}, \theta)}, n\right) \subset\left(\tilde{R}, p_{r}, \theta\right)_{n}$ with $\left(S_{(\tilde{R}, \theta)}, n\right)-\lim x=\left(\tilde{R}, p_{r}, \theta\right)_{n}-$ $\lim x=\xi$.

Proof Let $x$ be convergent to $\xi$ in $\left(S_{(\tilde{R}, \theta)}, n\right)$ and let us take

$$
K_{r m}(\varepsilon)=\left\{k \in I_{r}^{\prime}: p_{k}\left\|t_{k m}(x-\xi e), z_{1}, \ldots, z_{n-1}\right\| \geq \varepsilon\right\} .
$$

Since $p_{k}\left\|t_{k m}(x-\xi e), z_{1}, \ldots, z_{n-1}\right\| \leq M$ for all $k \in \mathbb{N}$ for each $m \geq 0$, for every nonzero $z_{1}, \ldots, z_{n-1} \in X$ and $H_{r} \rightarrow \infty$ as $r \rightarrow \infty$, then for a given $\varepsilon>0$ we have

$$
\begin{aligned}
\frac{1}{H_{r}} \sum_{k \in I_{r}} p_{k}\left\|t_{k m}(x-\xi e), z_{1}, \ldots, z_{n-1}\right\|= & \frac{1}{H_{r}} \sum_{\substack{k \in I_{r} \\
k \in K_{r m(\varepsilon)}}} p_{k}\left\|t_{k m}(x-\xi e), z_{1}, \ldots, z_{n-1}\right\| \\
& +\frac{1}{H_{r}} \sum_{\substack{k \in I_{r} \\
k \notin K_{r m(\varepsilon)}}} p_{k}\left\|t_{k m}(x-\xi e), z_{1}, \ldots, z_{n-1}\right\| \\
\leq & M \frac{1}{H_{r}}\left|K_{r m(\varepsilon)}\right|+\frac{h_{r}}{H_{r}} \varepsilon \\
\leq & M \frac{1}{H_{r}}\left|K_{r m(\varepsilon)}\right|+\varepsilon,
\end{aligned}
$$


for each $m \geq 0$ and for every nonzero $z_{1}, \ldots, z_{n-1} \in X$. Since $\varepsilon$ is arbitrary, we have $x \in$ $\left(\tilde{R}, p_{r}, \theta\right)_{n}$ by taking the limit as $r \rightarrow \infty$.

Theorem 3.9 The following statements are true.

(1) If $p_{k} \leq 1$ for all $k \in \mathbb{N}$, then $\left(S_{\theta}, n\right) \subseteq\left(S_{(\tilde{R}, \theta)}, n\right)$.

(2) Let $p_{k} \geq 1$ for all $k \in \mathbb{N}$ and $\left(\frac{H_{r}}{h_{r}}\right)$ be upper-bounded, then $\left(S_{(\tilde{R}, \theta)}, n\right) \subseteq\left(S_{\theta}, n\right)$.

\section{Proof}

(1) If $p_{k} \leq 1$ for all $k \in \mathbb{N}$, then $H_{r} \leq h_{r}$ for all $r \in \mathbb{N}$. So, there exist $M_{1}$ and $M_{2}$, constants, such that $0<M_{1} \leq \frac{H_{r}}{h_{r}} \leq M_{2} \leq 1$ for all $r \in \mathbb{N}$. Let $x \in\left(S_{\theta}, n\right)$ with $\left(S_{\theta}, n\right)-\lim x=\xi$, then for an arbitrary $\varepsilon>0$ we have

$$
\begin{aligned}
& \frac{1}{H_{r}}\left|\left\{k \in I_{r}^{\prime}: p_{k}\left\|t_{k m}(x-\xi e), z_{1}, \ldots, z_{n-1}\right\| \geq \varepsilon\right\}\right| \\
& \quad=\frac{1}{H_{r}}\left|\left\{P_{k_{r-1}}<k \leq P_{k_{r}}: p_{k}\left\|t_{k m}(x-\xi e), z_{1}, \ldots, z_{n-1}\right\| \geq \varepsilon\right\}\right| \\
& \quad \leq \frac{1}{M_{1}} \frac{1}{h_{r}}\left|\left\{P_{k_{r-1}} \leq k_{r-1}<k \leq P_{k_{r}} \leq k_{r}:\left\|t_{k m}(x-\xi e), z_{1}, \ldots, z_{n-1}\right\| \geq \varepsilon\right\}\right| \\
& \quad=\frac{1}{M_{1}} \frac{1}{h_{r}}\left|\left\{k_{r-1}<k \leq k_{r}:\left\|t_{k m}(x-\xi e), z_{1}, \ldots, z_{n-1}\right\| \geq \varepsilon\right\}\right| \\
& \quad=\frac{1}{M_{1}} \frac{1}{h_{r}}\left|\left\{k \in I_{r}:\left\|t_{k m}(x-\xi e), z_{1}, \ldots, z_{n-1}\right\| \geq \varepsilon\right\}\right|,
\end{aligned}
$$

for each $m \geq 0$ and for every nonzero $z_{1}, \ldots, z_{n-1} \in X$. Hence, we obtain the result by taking the limit as $r \rightarrow \infty$.

(2) Let $\left(\frac{H_{r}}{h_{r}}\right)$ be upper-bounded, then there exist $M_{1}$ and $M_{2}$, constants, such that $1 \leq M_{1} \leq \frac{H_{r}}{h_{r}} \leq M_{2}<\infty$ for all $r \in \mathbb{N}$. Suppose that $p_{k} \geq 1$ for all $k \in \mathbb{N}$, then $H_{r} \geq h_{r}$ for all $r \in \mathbb{N}$. Let $x \in\left(\tilde{R}, p_{r}\right)_{n}$ and $\left(\tilde{R}, p_{r}\right)_{n}-\lim x=\xi$, then for an arbitrary $\varepsilon>0$ we have

$$
\begin{aligned}
& \frac{1}{h_{r}}\left|\left\{k \in I_{r}:\left\|t_{k m}(x-\xi e), z_{1}, \ldots, z_{n-1}\right\| \geq \varepsilon\right\}\right| \\
& \quad=\frac{1}{h_{r}}\left|\left\{k_{r-1}<k \leq k_{r}:\left\|t_{k m}(x-\xi e), z_{1}, \ldots, z_{n-1}\right\| \geq \varepsilon\right\}\right| \\
& \quad \leq M_{2} \frac{1}{H_{r}}\left|\left\{k_{r-1} \leq P_{k_{r-1}}<k \leq k_{r} \leq P_{k_{r}}: p_{k}\left\|t_{k m}(x-\xi e), z_{1}, \ldots, z_{n-1}\right\| \geq \varepsilon\right\}\right| \\
& \quad=M_{2} \frac{1}{H_{r}}\left|\left\{P_{k_{r-1}}<k \leq P_{k_{r}}: p_{k}\left\|t_{k m}(x-\xi e), z_{1}, \ldots, z_{n-1}\right\| \geq \varepsilon\right\}\right| \\
& \quad=M_{2} \frac{1}{H_{r}}\left|\left\{k \in I_{r}^{\prime}: p_{k}\left\|t_{k m}(x-\xi e), z_{1}, \ldots, z_{n-1}\right\| \geq \varepsilon\right\}\right|,
\end{aligned}
$$

for each $m \geq 0$ and for every nonzero $z_{1}, \ldots, z_{n-1} \in X$. Hence, the result is obtained by taking the limit as $r \rightarrow \infty$.

Theorem 3.10 For any lacunary sequence $\theta$, if $\liminf _{r} Q_{r}>1$ then $\left(S_{\tilde{R}}, n\right) \subseteq\left(S_{(\tilde{R}, \theta)}, n\right)$ and $\left(S_{\tilde{R}}, n\right)-\lim x=\left(S_{(\tilde{R}, \theta)}, n\right)-\lim x=\xi$.

Proof Suppose that $\liminf _{r} Q_{r}>1$, then there exists a $\delta>0$ such that $Q_{r} \geq 1+\delta$ for sufficiently large values of $r$, which implies that $\frac{H_{r}}{P_{k_{r}}} \geq \frac{\delta}{1+\delta}$. If $x \in\left(S_{\tilde{R}}, n\right)$ with $\left(S_{\tilde{R}}, n\right)-\lim x=\xi$, 
then for every $\varepsilon>0$ and for sufficiently large values of $r$, we have

$$
\begin{aligned}
& \frac{1}{P_{k_{r}}}\left|\left\{k \leq P_{k_{r}}: p_{k}\left\|t_{k m}(x-\xi e), z_{1}, \ldots, z_{n-1}\right\| \geq \varepsilon\right\}\right| \\
& \quad \geq \frac{1}{P_{k_{r}}}\left|\left\{P_{k_{r-1}}<k \leq P_{k_{r}}: p_{k}\left\|t_{k m}(x-\xi e), z_{1}, \ldots, z_{n-1}\right\| \geq \varepsilon\right\}\right| \\
& \quad=\frac{H_{r}}{P_{k_{r}}}\left(\frac{1}{H_{r}}\left|\left\{P_{k_{r-1}}<k \leq P_{k_{r}}: p_{k}\left\|t_{k m}(x-\xi e), z_{1}, \ldots, z_{n-1}\right\| \geq \varepsilon\right\}\right|\right) \\
& \quad \geq \frac{\delta}{1+\delta}\left(\frac{1}{H_{r}}\left|\left\{k \in I_{r}^{\prime}: p_{k}\left\|t_{k m}(x-\xi e), z_{1}, \ldots, z_{n-1}\right\| \geq \varepsilon\right\}\right|\right),
\end{aligned}
$$

for each $m \geq 0$ and for every nonzero $z_{1}, \ldots, z_{n-1} \in X$. Hence, we get the result by taking the limit as $r \rightarrow \infty$.

Theorem 3.11 Let $\theta=\left(k_{r}\right)$ be a lacunary sequence with $\lim \sup _{r} Q_{r}<\infty$, then $\left(S_{(\tilde{R}, \theta)}, n\right) \subseteq$ $\left(S_{\tilde{R}}, n\right)$ and $\left(S_{\tilde{R}}, n\right)-\lim x=\left(S_{(\tilde{R}, \theta)}, n\right)-\lim x=\xi$.

Proof If $\lim \sup _{r} Q_{r}<\infty$, then there is a $K>0$ such that $Q_{r} \leq K$ for all $r \in \mathbb{N}$. Suppose that $x \in\left(S_{(\tilde{R}, \theta)}, n\right)$ with $\left(S_{(\tilde{R}, \theta)}, n\right)-\lim x=\xi$ and let

$$
N_{r}:=\left|\left\{k \in I_{r}^{\prime}: p_{k}\left\|t_{k m}(x-\xi e), z_{1}, \ldots, z_{n-1}\right\| \geq \varepsilon\right\}\right| .
$$

By (3.7), given $\varepsilon>0$, there is a $r_{0} \in \mathbb{N}$ such that $\frac{N_{r}}{H_{r}}<\varepsilon$ for all $r>r_{0}$. Now, let $M:=$ $\max \left\{N_{r}: 1 \leq r \leq r_{0}\right\}$ and let $r$ be any integer satisfying $k_{r-1}<r \leq k_{r}$, then for each $m \geq 0$ and for every nonzero $z_{1}, \ldots, z_{n-1} \in X$ we can write

$$
\begin{aligned}
\frac{1}{P_{r}} & \left|\left\{k \leq P_{r}: p_{k}\left\|t_{k m}(x-\xi e), z_{1}, \ldots, z_{n-1}\right\| \geq \varepsilon\right\}\right| \\
& \leq \frac{1}{P_{k_{r-1}}}\left|\left\{P_{k_{r-1}}<k \leq P_{k_{r}}: p_{k}\left\|t_{k m}(x-\xi e), z_{1}, \ldots, z_{n-1}\right\| \geq \varepsilon\right\}\right| \\
& =\frac{1}{P_{k_{r-1}}}\left(N_{1}+N_{2}+\cdots+N_{r_{0}}+N_{r_{0}+1}+\cdots+N_{r}\right) \\
& \leq \frac{M . r_{0}}{P_{k_{r-1}}}+\frac{1}{P_{k_{r-1}}} \varepsilon\left(H_{r_{0}+1}+\cdots+H_{r}\right) \\
& =\frac{M \cdot r_{0}}{P_{k_{r-1}}}+\varepsilon \frac{\left(P_{k_{r}}-P_{k_{r_{0}}}\right)}{P_{k_{r-1}}} \\
& \leq \frac{M . r_{0}}{P_{k_{r-1}}}+\varepsilon Q_{r} \leq \frac{M \cdot r_{0}}{P_{k_{r-1}}}+\varepsilon K,
\end{aligned}
$$

which completes the proof by taking the limit as $r \rightarrow \infty$.

Corollary 3.12 Let $1<\liminf _{r} Q_{r} \leq \lim \sup _{r} Q_{r}<\infty$. Then $\left(S_{(\tilde{R}, \theta)}, n\right)=\left(S_{\tilde{R}}, n\right)$ and $\left(S_{\tilde{R}}, n\right)-$ $\lim x=\left(S_{(\tilde{R}, \theta)}, n\right)-\lim x=\xi$.

Proof It follows from Theorem 3.10 and Theorem 3.11. 


\section{Competing interests}

The authors declare that they have no competing interests.

\section{Authors' contributions}

All authors contributed equally in the preparation of this article. Both authors read and approved the final manuscript.

\section{Acknowledgements}

This paper has been presented in 2nd International Eurasian Conference on Mathematical Sciences and Applications

(IECMSA-2013) and it was supported by the Research Foundation of Sakarya University (Project Number: 2012-50-02-032)

\section{Received: 4 October 2013 Accepted: 9 January 2014 Published: 18 Feb 2014}

\section{References}

1. Gähler, S: Lineare 2-normierte raume. Math. Nachr. 28, 1-43 (1965)

2. Misiak, A: n-Inner product spaces. Math. Nachr. 140, 299-319 (1989)

3. Gürdal, M, Pehlivan, S: Statistical convergence in 2-normed spaces. Southeast Asian Bull. Math. 33, 257-264 (2009)

4. Başarır, M, Konca, Ş, Kara, EE: Some generalized difference statistically convergent sequence spaces in 2-normed space. J. Inequal. Appl. 2013(177), 1-12 (2013)

5. Başarır, M, Konca, Ş: Some sequence spaces derived by Riesz mean in a real 2-normed space. Iran. J. Sci. Technol., Trans. A, Sci. Accepted

6. Gunawan, H, Mashadi, M: On n-normed spaces. Int. J. Math. Math. Sci. 27(10), 631-639 (2001)

7. Gunawan, H: The space of p-summable sequences and its natural n-norm. Bull. Aust. Math. Soc. 64, 137-147 (2001)

8. Gunawan, H, Setya-Budhi, W, Mashadi, M, Gemawati, S: On volumes of $n$-dimensional parallelepipeds in $\mathbb{P}$ spaces. Publ. Elektroteh. Fak. Univ. Beogr., Mat. 16, 48-54 (2005)

9. Reddy, BS: Statistical convergence in $n$-normed spaces. Int. Math. Forum 5, 1185-1193 (2010)

10. Konca, Ş, Başarır, M: Generalized difference sequence spaces associated with a multiplier sequence on a real n-normed space. J. Inequal. Appl. 2013(335), 1-18 (2013)

11. Zygmund, A: Trigonometric Spaces. Cambridge University Press, Cambridge (1979)

12. Fast, H: Sur la convergence statistique. Colloq. Math. 2(3-4), 241-244 (1951)

13. Steinhaus, H: Sur la convergence ordinate et la convergence asymptotique. Colloq. Math. 2, $73-84$ (1951)

14. Schoenberg, IJ: The integrability of certain functions and related summability methods. Am. Math. Mon. 66, 361-375 (1959)

15. Buck, RC: Generalized asymptotic density. Am. J. Math. 75, 335-346 (1953)

16. Fridy, JA, Orhan, C: Lacunary statistical convergence. Pac. J. Math. 160, 43-51 (1993)

17. Moricz, F, Orhan, C: Tauberian conditions under which statistical convergence follows from statistical summability by weighted means. Studia Sci. Math. Hung. 41, 391-403 (2004)

18. Karakaya, V, Chishti, TA: Weighted statistical convergence. Iran. J. Sci. Technol., Trans. A, Sci. 33(A3), $219-223$ (2009)

19. Mursaleen, M, Karakaya, V, Ertürk, M, Gürsoy, F: Weighted statistical convergence and its application to Korovkin type approximation theorem. Appl. Math. Comput. 218, 9132-9137 (2012)

20. Başarır, M, Öztürk, M: On the Riesz difference sequence space. Rend. Circ. Mat. Palermo 57, 377-389 (2008)

21. Başarır, M, Kara, EE: On compact operators on the Riesz difference sequence spaces. Iran. J. Sci. Technol., Trans. A, Sci. 35(A4), 279-285 (2011)

22. Başarır, M, Kara, EE: On compact operators on the Riesz difference sequence spaces II. Iran. J. Sci. Technol., Trans. A, Sci. 4, 371-376 (2012)

23. Başarır, M, Kayıkçı, M: On the generalized Riesz difference sequence space and property. J. Inequal. Appl. 2009, Article ID 385029 (2009). doi:10.1155/2009/385029

24. Başarır, M, Konca, Ş: On some spaces of lacunary convergent sequences derived by Norlund-type mean and weighted lacunary statistical convergence. Arab J. Math. Sci. (2013, in press)

25. Lorentz, GG: A contribution to the theory of divergent sequences. Acta Math. 80(1), 167-190 (1948)

26. Maddox, IJ: A new type of convergence. Math. Proc. Camb. Philos. Soc. 83, 61-64 (1978)

10.1186/1029-242X-2014-81

Cite this article as: Konca and Başarır: On some spaces of almost lacunary convergent sequences derived by Riesz mean and weighted almost lacunary statistical convergence in a real $n$-normed space. Journal of Inequalities and Applications 2014, 2014:81 